\title{
LEARNING PATIENT ADHERENCE FROM THE CLINICAL NARRATIVES: A THICK DATA APPROACH FOR VALUE BASED HEALTHCARE
}

\author{
Sabah Mohammed ${ }^{1}$, Jinan Fiaidhi ${ }^{2}$ and Sami Mohammed ${ }^{3 *}$ \\ ${ }^{1}$ Department of Computer Science, Lakehead University, Canada \\ ${ }^{2}$ Department of Computer Science, Lakehead University, Canada \\ ${ }^{3}$ Department of Computer Science, University of Victoria, Canada \\ ${ }^{1}$ sabah.mohammed@lakeheadu.ca, ${ }^{2}$ jfiaidhi@lakeheadu.ca, ${ }^{3}$ smohamm@uvic.ca
}

Abstract - This paper presents a practice-based conceptual (theoretical) model to address therapy adherence. The paper uses the new notion of big data to describe of a model that can provide qualitative values for patient adherence and insights from the clinical narratives. This model has been designed to provide clinicians with a new approach to support the value based healthcare initiatives. The model is not fully tested as it is the next phase of the authors work.

Keywords - Thick Data, Medication Adherence, Clinical Narratives, Patient Insight

\section{TOWARDS VALUE-BASED HEALTHCARE:}

Value-based healthcare is a new healthcare delivery model in which providers, including hospitals and physicians, are paid based on patient health outcomes. It is different from the current healthcare system that is based on fee-for-service to reimburse health providers for individual services. The fee-for-service model is widely indicted for promoting care that is inefficient, uncoordinated, and too often fails to meet the needs of patients and improving the health and wellbeing outcomes. The "value-based" have thus become the new buzz words for payment and delivery reform in health care. However, everyone knows that the pathway to value based healthcare is a big challenge and will not be achieved through adding more regulations and policies as administrators has appropriately recognized that an over-regulation will impede the transition to a truly value-based health care system. Figure 1 illustrates the three major parameters that can contribute to a value based healthcare.

Received: November 26, 2018

Reviewed: January 28, 2019

Accepted: February 4, 2019

* Corresponding Author 


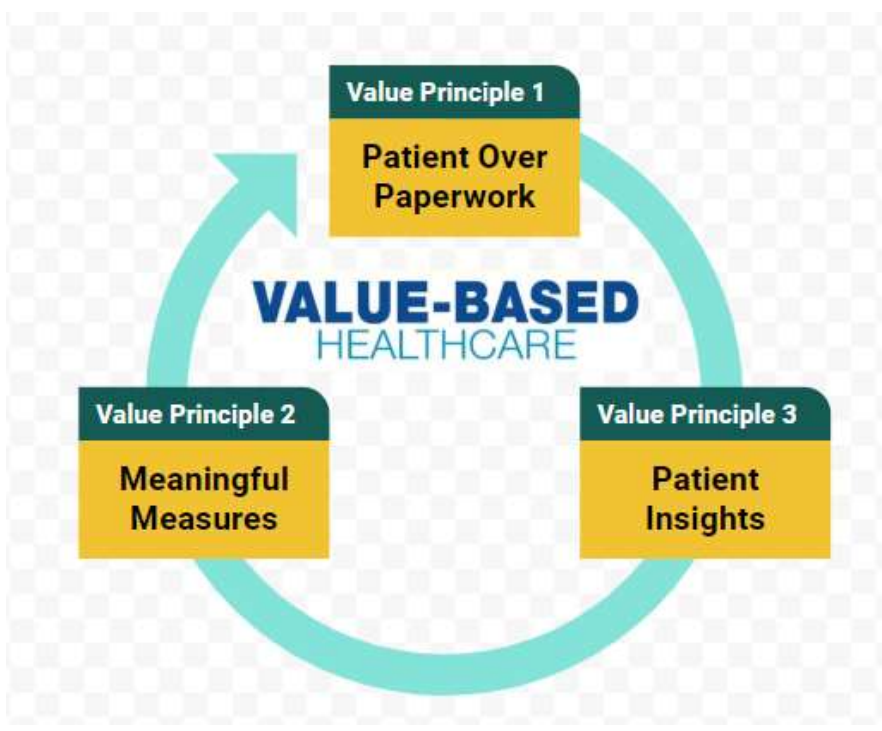

Fig. 1 The Three Most Important Principles for the Value Based Healthcare.

The "Meaningful Measures" framework is the principle which identifies the highest priorities for quality measurement and improvement. It involves only assessing those core issues that are the most critical to providing high-quality care and improving individual outcomes. The Meaningful Measure Areas serve as the connectors between the education strategic goals and measures that demonstrate how high quality outcomes for care are being achieved. They are concrete quality topics, which reflect core issues that are most vital to high quality care and better patient outcomes. The "Patients over Paperwork" principle is to reduce burdensome regulations and increase efficiencies as well as to improve the beneficiary experience. In carrying out this principle, the healthcare system is moving the needle and removing regulatory obstacles that get in the way of health providers spending time with patients. "Patient Insights" principle advocates for the process of engaging with the patient in order to gaining an in-depth understanding of the patient and the many factors that influence their health behavior and decision-making process. The goal is to bring the patient's perspective into all aspects and phases of the health evaluation process.

The general believe has been always to rely on Big Data and predictive analytics to make relevant, personalized, and precisely timed offers to customers. However, using such approach proves to be daunting if it is built on prediction models that are based on quantitative datasets and do not incorporate important consumer values and insights. According to Tricia Wang [1] "What is measurable isn't the same as what is valuable". There are many alarming examples that proves the failure of using pure quantitative consumer data to derive business decisions like the Nokia failure although Nokia have collected huge amount of data about their customers and the market but failed to alert the company about the consumer's values and the market trends for the smartphone technology [2]. Many missing factors are associated with using general quantitative data to tap on patient insights. It includes the power to understand the nuances of patient behavior with the best-in-class insights technologies.

From the above discussion we know that big data is not working. Thick data on the other hand which is a concept coined by Tricia Wang puts more weight on the qualitative data to provide the context that enables the business to understand consumer insights. Based on Thick data Analytics, we can understand the quirks of patient behavior to predict how an individual's relationship with the health service or medication will evolve over time. Without this understanding, quantitative patterns that the business may uncover that suggest patients will behave in a certain way could be based in a world that 
no longer exists. Figure 2 illustrate the general view of the new value-based healthcare that uses more Thick Data analytics than Big Data analytics.

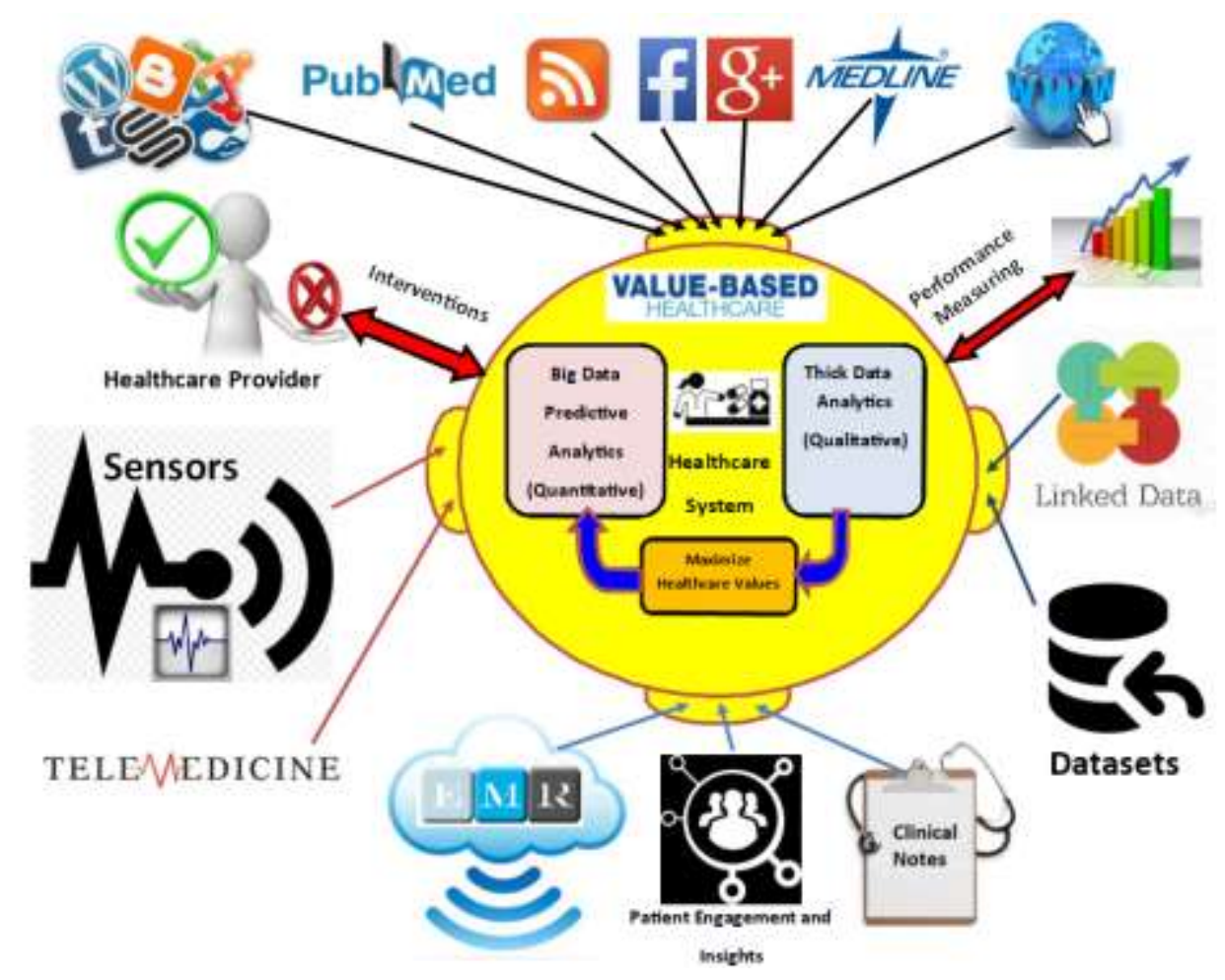

Fig. 2 The Overall View of a Value Based Healthcare System

\section{THICK DATA ANALYTICS: COMBING PATIENT INSIGHTS WITH ADHERENCE}

Thick data analytics may use variety of qualitative techniques to tap on consumer insights and measure the adherence to the medical instructions. For example, in analyzing data that included prescription or medication there are many factors that affect patient satisfaction including drug-related characteristics, factors related to the condition itself, healthcare-system factors, and sociodemographic and patient-related factors. Without understanding all these factors, we cannot fully decode the values behind prescribing a drug for a patient and interpreting properly the patient satisfaction on this particular drag. The part related to patient insights can be valued using several techniques:

> Patient Reviews and Complaints - Reading patients reviews can highlight common problems or wishes. Patient feedback is an opportunity to make the healthcare system better. Each individual patient complaint potentially represents a much larger sentiment. Therefore, it's worthwhile to understand your patient' experience and identify areas for improvement of the system.

$>$ Q\&A sites - These sites can give you an idea of the questions and concerns that patients have in relation to you're a medication, health service service or product.

$>$ Surveys - Online surveys can be easily set up with sites like Survey Monkey and allow you to ask specific questions.

$>$ Focus groups - Bring a group of patients together and ask them questions directly. 
Keyword research - A mainstay of SEO, keyword research can tell you what patients are interested in and the relative level of interest. It also helps to reveal the language they are using.

$>$ Google Analytics - Analytics can be used to tell you where your traffic is coming from. The Audience tab shows geography, interests, and a range of demographics. Google analytics can help you understand patient behavior

> Blog comments - Comments on your health blog can be a good way of discovering any questions your patients might have.

$>$ Twitter Insiders - Twitter recently launched Insiders, a 12,000 strong focus group of US \& UK Twitter users.

$>$ Google trends - Google Trends can help you to understand if a topic is becoming more or less popular.

$>$ Government data - Government data is available for free and can help you understand a group, and several other sources can also be accessed without charge.

> Social media - Millions of people reflect their lives on social media, so information that can enrich several strands of consumer behavior can be uncovered with the right tools.

However, knowing the patient adherence to treatment or a therapy is essential to optimize disease management and provide better patient satisfaction. As with many chronic diseases, poor adherence is common and results in increased rates of morbidity, healthcare expenditures, hospitalizations and possibly mortality, as well as unnecessary escalation of therapy and reduced quality of life. Examples poor adherence include overuse, underuse, and alteration of schedule and doses of medication, continued smoking and lack of exercise. Adherence is affected by patients' perception of their disease, type of treatment or medication, the quality of patient provider communication and the social environment. Patients are more likely to adhere to treatment when they believe it will improve disease management or control, or anticipate serious consequences related to non-adherence. Providers play a critical role in helping patients understand the nature of the disease, potential benefits of treatment, addressing concerns regarding potential adverse effects and events, and encouraging patients to develop selfmanagement skills. For clinicians, it is important to explore patients' beliefs and concerns about the safety and benefits of the treatment, as many patients harbor unspoken fears. Complex regimens and polytherapy also contribute to suboptimal adherence. Many thick data techniques can be employed to explore patient adherence. However, the clinical narrative provided by the physician to the patient is one of the most important source of knowledge to the patient adherence of the prescribed therapy.

\section{QUALIFYING ADHERENCE TO A THERAPY FROM THE CLINICAL NARRATIVE}

There are a number of ways to measure adherence, and each method has strengths and limitations. Most studies focused on assessing medication adherence. Biochemical evaluation of drug levels can confirm ingestion of many medications. However, it is an expensive and invasive process and often reflects other factors resulting in pharmacokinetic variations. Electronic medical devices can provide objective evidence of the temporal history of medication access. For instance, patients' pill bottles can be equipped with caps that provide a date and time record of each time the cap was removed. Cap removal suggests (but does not confirm) medication ingestion. Chronologs can be 
attached to metered dose inhalers to provide a record of dosing and, in some cases, even give an indication of the quality of inhaling technique. Electronic monitors are increasingly used in clinical trials to measure adherence. They provide accurate and reliable records of dosing but are expensive to use, subject to malfunction and cannot usually confirm ingestion. However, studying clinical narrative provide valuable information on the patient story and the reaction to the therapies and medications provided including many hints to adherence. From the medical perspective reaching the accurate diagnosis is often the end of story which provide relief to patients; but Illness continues to unfold over time. In other words the patient's history is provisional and continuously changes. This is of particular importance as we provide care to the elderly, many of whom have complex, chronic health problems. The patient's narrative provides meaning, context, and an understanding of the patient's experience of illness. In other words, the narrative builds the bridge connecting the evidence of large clinical trials to the individual patient. The complete story from the perspective of both the doctor and the patient increases diagnostic accuracy, and better informs us as to whether an evidencebased intervention applies to the patient before you. The fear often expressed by physicians is not having enough time to hear the patient's story. However, allowing for the narrative to unfold does NOT always require more time.

Actually, no practical techniques exist for a large-scale assessment of the nonadherence phenomenon. However, the clinical narrative and notes provide many phrases and words that indicate non-compliance like:

noncompliance

\section{disregard}

inobservance

omission

failure

oversight

dereliction

nonfeasance

slight

nonconformity

neglect

laxity

negligence

inattention

nonfulfillment

nonperformance

underperformance

delinquency

default

Non-compliant,

reluctant

did not wish 


\author{
does not want \\ declines \\ declined \\ refuses \\ refused \\ has not been taking \\ has not taken \\ not interested \\ Did not show \\ Did not come \\ DNKA \\ no-show, \\ no showed \\ did not keep his last appointment \\ did not keep your appointment \\ missed the last appointment
}

In [3] a group of physicians from Brigham and Women's Hospital developed a computational approach that quantifies patient non-adherence from an analysis of the text of physician notes. Index of non-adherence (INA) was computed based on the number of non-adherence word tags detected in physician notes. INA was evaluated by comparing the results to a manual patient record review at the individual sentence and patient level. The relationship between INA and frequency of Emergency Department visits was determined. The positive predictive value of identification of individual non-adherence word tags was $93.3 \%$. The Pearson correlation coefficient between the INA and the number of documented instances of non-adherence identified by manual review was 0.62. The frequency of ED visits was more than twice as high for patients with INA in the highest quartile (least adherent) than for patients with INA in the lowest (most adherent) quartile ( $p$ 50.0001). We have described the design and evaluation of a novel approach that allows quantification of patient nonadherence with physician recommendations through an analysis of physician notes. This approach has been validated at several levels and demonstrated to correlate with clinical outcomes. Figure 3 illustrates the steps for combining non-adherence data and patient insights to create a thick data report on the care value. 


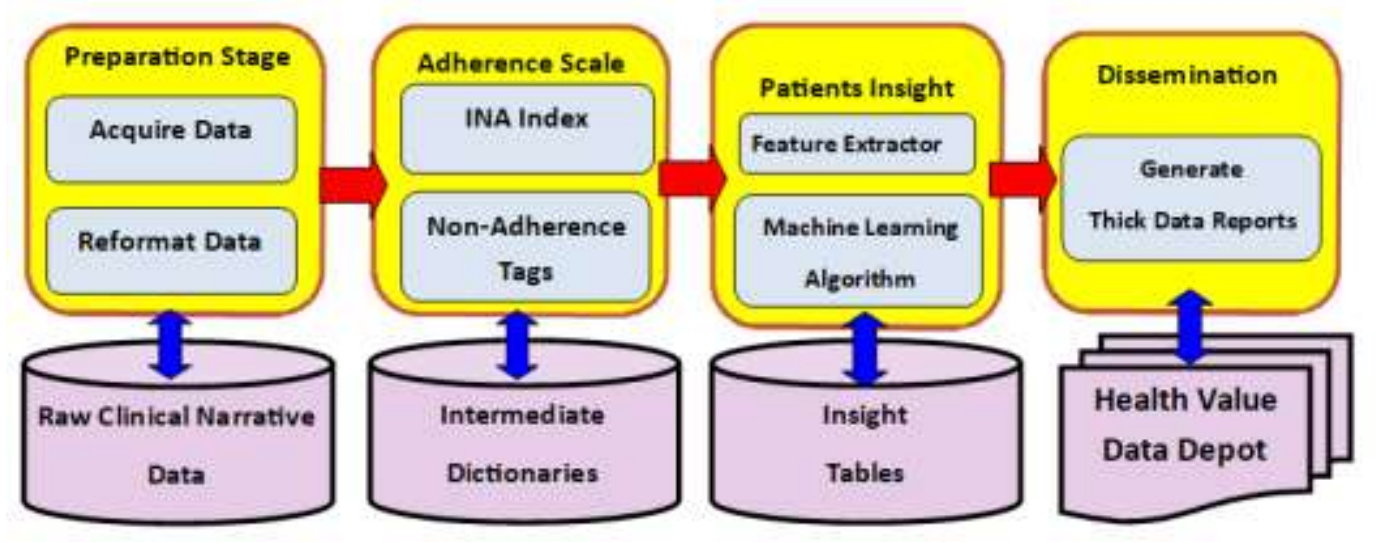

Fig. 3 A Platform for Evaluating Health Values from the Clinical Narratives.

The authors of this paper is in the process now to test the above platform on the i2b2 dataset $^{1}$ in combinations with patient data for similar cases from popular patients blogs like patient_like-me ${ }^{2}$ and drugs.com. ${ }^{3}$

\section{CONCLUSIONS}

The term adherence, which initially referred solely to adherence to a treatment regimen, has evolved to reflect the changing perceptions of a patient's role in their own healthcare. The primary difference between "compliance" and the "adherence" is that the latter more accurately reflects a patient's active role in consenting to and following prescribed treatments. The objective of this article is to create a practical thick data analysis framework to analyze clinical narratives healthcare data like discharge summaries and the patient narratives on the notable online blogs to provide qualitative image on patient adherence and insights as indicators for the value of care and treatment.

\section{REFERENCES}

[1] Wang, Tricia, Why Big Data Needs Thick Data, Medium.com blog, Jan 20, 2016, Available Online: https://medium.com/ethnography-matters/why-big-data-needs-thick-data-b4b3e75e3d7

[2] BRAND MINDS, Why did Nokia fail and what can you learn from it? Jul 24, 2018, Available online: https://medium.com/multiplier-magazine/why-did-nokia-fail-81110d981787

[3] ALEXANDER TURCHIN, NIKHEEL S. KOLATKAR, MERRI L. PENDERGRASS and ISAAC S. KOHANE, Computational analysis of non-adherence and non-attendance using the text of narrative physician notes

\footnotetext{
${ }^{1}$ https://www.i2b2.org/NLP/DataSets/

${ }^{2}$ https://www.patientslikeme.com/

${ }^{3} \mathrm{https}: / /$ www.drugs.com/
} 
International Journal of Advanced Science and Technology Vol.124 (2019) 